\title{
AN EXPERIMENTAL SOLUTION FOR SYSTEMATIC BROADCASTING DATA ADOPTING FORWARDER NODES INFORMATION WITH IT VANETS
}

\author{
S. Karimulla Basha \\ Research Scholar, Department of computer Science and engineering \\ Koneru Lakshmaiah Education Foundation, Vaddeswaram, \\ Guntur, A.P., India -522022 \\ kareem768@gmail.com \\ T. N. Shankar \\ Professor, Department of computer Science and engineering \\ Koneru Lakshmaiah Education Foundation, Vaddeswaram, \\ Guntur, A.P., India -522022 \\ tnshankar2004@kluniversity.in
}

\begin{abstract}
From the movement of vehicle and wireless channels likes lossy, challenging an efficient and reliable multi-hop broadcast protocol service in VANETs (Vehicular Ad hoc Networks). In this paper, main task is multi-hop broadcast protocol using a fuzzy logic based method in VANETs. The proposed protocol is FuzzyBR-FNI (Fuzzy broadcast with forwarder node information) and obtains a low overhead by utilizing a subset of forwarder nodes to neighbor data packets. Considering the forwarder node selection, FuzzyBR-FNI together with more than one metrics of the inter-vehicle distance, vehicle mobility and Received Signal Strength Indication by utilizing fuzzy logic technique. After all the expected coverage distance and vehicle mobility are assumed from the forwarder node information which is obtained from the emergency message exchange. FuzzyBR-FNI provides an experimental and convenient result for broadcast facilities in VANETs because FuzzyBR-FNI is independent of position information. We employ real-world practicals and computer simulations to evaluate the performance of FuzzyBR-FNI. The method appraisal is skilled in veins tools and MATLAB environment to prove the efficiency of it.
\end{abstract}

Keywords: fuzzy logic; broadcast protocol; forwarder information; Vehicular Ad hoc Networks; experimental solution.

\section{Introduction}

A (VANET) Vehicular Ad hoc Network is used to establish communication among vehicles in close contiguity, among vehicles and nearness fixed roadside device. A multi-hop broadcast protocol is obtained to disseminate traffic warning information (inter-vehicle communication warning, work zone warning etc) to more than one receivers in VANETs. Decreasing broadcast redundancy is essential since it can face to face affect MAC layer contention time at every one node and work on the packet collision probability. In VANETs broadcast protocols are classified to (a) receiver-oriented protocols and (b) sender-oriented protocols. Above the reception of the packets, in the receiver-oriented protocols each node chooses whether to rebroadcast a packet or not. The sender vehicle represents the next forwarders in the sender-oriented protocols. Some of the receiver -oriented protocols are available at [1]-[6] and the redundant broadcasts cannot be excluded absolutely. The sender node describes the forwarder nodes, in sender-oriented protocols. Generally, the selection of forwarder nodes $\dot{\mathbf{s}}$ placed on the message collected by exchanging emergency messages between neighbor nodes. Consequently, for sender oriented protocols the selection of forwarder node algorithm is explicitly an essential part. In existing tasks [7],[8] selecting the efficient forwarder nodes and these are employed position message and road maps which are in accessible in some roads such as lanes. By using Multi-Point Relay (MPR) vehicles to forward 
data packets [9].These are having multiple increased nearer [10],[11].Though, these approaches are not providing link quality in forwarder node selection. As a result, researchers cannot perform thoroughly in fading surroundings. We introduce multi-hop broadcast protocol in a VANET which is free of location information in this research work. The proposed protocol chooses efficient forwarder nodes by using neighbor information which is collected from emergency message swaps in the neighborhood.

For one and all forwarder node candidate, to evaluate the inter-vehicle distance (for each one-hop neighbor) and node variation the proposed protocol (FuzzyBR-FNI) uses more than one hop neighbor information. T o evaluate the RSSI (Received Signal Strength Indication) between more than one neighbor, the emergency message reception ratio is utilized. The inter-vehicle distance, vehicle mobility and RSSI for the selection of forwarder node is considered in the proposed protocol utilizes a fuzzy logic based method. For further enhancement of reliability, the protocol also uses a common redundancy and greedy transmission approach. By rectifying the fuzzy logic membership functions, the protocol used is modulated for use in different scenarios. Therefore, the protocol can establish a smooth and mobile solution for more than one hop broadcast in VANETs. Using computer simulations and real-world analysis we appraise the proposed protocol by comparing it with alternatives. Veins software is used to develop this research paper in VANETs.

The organization of the research work is provided as follows. In sec. 2, we specify a skimpy form of related works. In sec. 3 we represent a full description of the proposed protocol, fuzzification. Following, we evaluate the Performance of analysis, clarify experimental results and simulation results in sec. 4 and sec. 5 respectively. Finally conclusions in sec. 6 .

\section{Related Work}

Several authors [1-4] propose various broadcasting protocols based on receivers. All these protocols make a precise decision for a node to broadcast further or stand still. Redundant broadcasting still exist and is not eliminated. These protocols cannot promise reliable transmission as probabilistic method in a sparse network.

The dissemination ratio varies in node selection of relay algorithm in most of the sender based protocols. An emergency broadcasting protocol was proposed in [7] by Sa-hoo et al. named as Binary Partition Assisted emergency Broadcast protocol (BPAB). Packets are relayed to the longest distant node in required direction. But due to severe channel fading, the longest transmission results in packet loss. Hence it is essential to consider the channel quality while designing a protocol for VANETs. One our previous work [8] opts a suitable relay node based on vehicular distance, movement and signal level using fuzzy logic. However, the works in [7],[8] emphasizes on position information of the node.

Few position independent work exist in the literature. A Multi-Point Relay (MPR) broadcast scheme in [9] was proposed by TQayyum et al. A broadcast protocol that opts relay nodes using Dominating Connected Set was proposed by Djedid et al. [10].However, Ref. [9] and Ref. [10] do not consider node mobility factor in selecting the appropriate relay node. As a result due to the node movement, the data packets can be lost at relay nodes. Relay nodes are selected by EMPR (Enhanced MPR Broadcast) [11] and the increased radio range and node mobility are observed. However, the wireless channels fading feature does not consider EMPR. A node could receive a hello message from a neighbor at a long distance in wireless channels where suitable conditions are not possible. The neighbor node fails to receive the packet with a high probability if a longdistance neighbor node is selected as a relay node. To solve this problem without deriving the mathematical model, we acquire a flexible solution which is based on fuzzy logic [12] approach. Simulation parameters, its environments and simulation results are used in [13-16]. A new technology in which detection of potential alarming environments are developed by the researcher by using basic messages to avoid accidental situations existed with a peak, iffy traffic conditions and to aid in reducing the broadcast messages [17-18].

Fig. 1 represents the four lane VANETs architecture which uses moving vehicles as nodes for communication. When any two vehicles are travelled for some distance then they get accidents, at that time source vehicle forward the emergency message to incoming of vehicles. Priority is giving which vehicle has long distance and maximum speed and less density such type of vehicle receives the emergency message. the four lane VANETs architecture which uses moving vehicles as nodes for communication. Nodes should be between 100 to 300 meters range. Communication can be between moving vehicles or any base stations. Drivers are alarmed of different road conditions. Communication between cars and road side can be performed by VANET. In this Fig. 1 it uses infrastructure, real time, crucial effect of security and privacy, time-sensitive data exchange as Potential support.

A potential dangerous situation on the road is the Emergency Messages which messages are sent by a vehicle detect. Vehicles are travelling with speed of $120 \mathrm{Km} /$ hour. The maximum range is $1 \mathrm{Km}$. Because of radiation effect and congestion road condition, it is not possible to forward the emergency message at a time to the incoming vehicles which are $1 \mathrm{Km}$ apart from source node. So this architecture is designed in such a way 
that emergency message gets forwarded for every $300 \mathrm{mts}$ to the destination node.

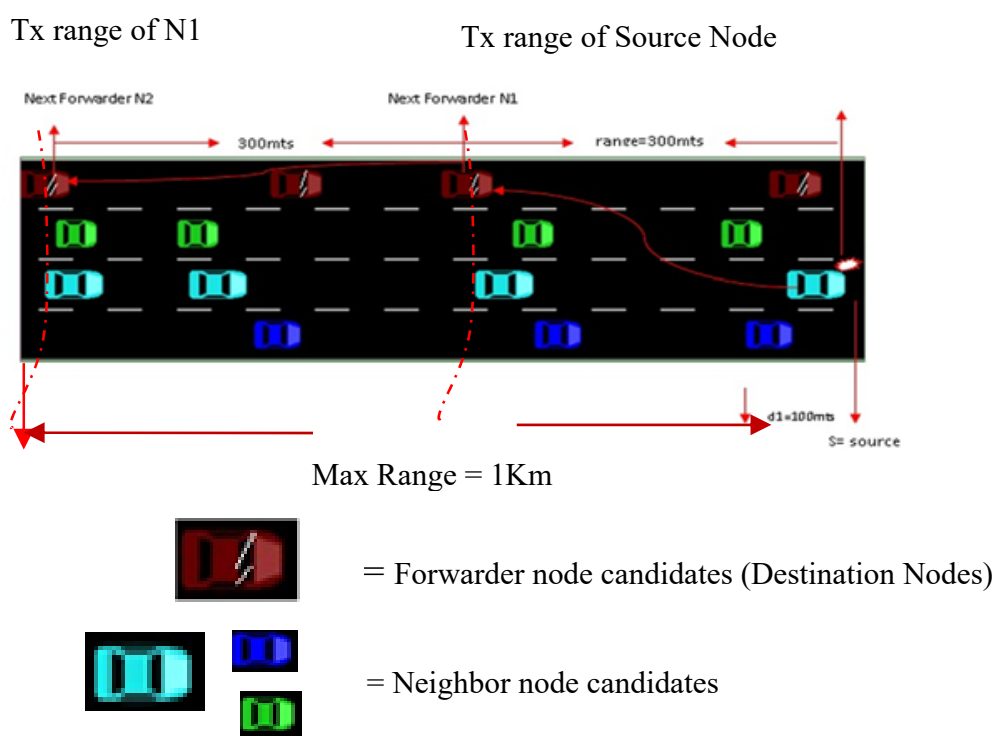

Fig. 1. Four Lanes Architecture in VANET

\section{Proposed Protocol: FuzzyBR-FNI}

\subsection{Protocol Design}

The FuzzyBR-FNI is a proposed protocol, it describes forwarder nodes to forward a packet. Each node occupies the address of the forwarder nodes to the packet prior to broadcasting a packet. Consequent to reception of a packet, a node can rebroadcast the packets specifically assuming it is on its own incorporated in the forwarder node list by lying on reception of a packet.

In the fuzzy logic broadcast forwarder node information (FuzzyBR-FNI), we provide vehicle mobility, inter-vehicle distance and RSSI. In order to provide a convenient response that is indifferent of position information, proposed protocol utilizes neighbor information to understand the vehicle mobility and intervehicle distance. In this paper, to represent one-hop neighbor node we specify "neighbor node", and employ " more than one hop neighbor node". Each node represents cannot be directly communicate but a node forwarding the packets with the help of another neighbor node.

VANET is a sharp network in which vehicles can exchange data while moving on roads or communicate with each other. In each vehicle, these networks have a wireless transceiver embedded in it which enables the vehicles to exchange data even when communication architecture is not presented. In FuzzyBR-FNI, each source node neighbor information (more than one hop) is used to evaluate forwarder node candidates set by fuzzy logic based method, we can select the best forwarder nodes in VANETs FuzzyBR-FNI also uses a designing transmission approach and link redundancy to enhance trust ability. The source node transmits a packet synergic times to establish a high reliability if the signal strength to a forwarder node is delicate. An adaptable transmission is utilized when a packet absence occurs at a forwarder node.

Based upon fuzzy set theory, fuzzy logic is agreed with the concept of approximate rather than exact. Elements have degrees of membership in the Fuzzy set theory which are different from classical set theory. Fuzzy set theory represents imprecise information or incomplete by defining set membership as a possibility distribution. By using non-numeric linguistic variables Fuzzy logic can process approximate data bexpress the facts. To represent the degrees of a numerical value belonging to predefined linguistic variables, Fuzzy membership functions are used. To conduct the final fuzzy value from the fuzzy, Fuzzy rules are defined. The final fuzzy value is converted to a numerical value by using Defuzzification.

\subsection{Forwarder Node Calculation Based on Fuzzy Logic}

\subsubsection{Fuzzy set theory and Fuzzy logic}

In this all the elements possess degrees of membership. Fuzzy logic refers if/then rule to represent with the fuzzy thesis. 


\subsubsection{Fuzzification}

Conversion of a numerical to fuzzy value by the membership function is known as 'fuzzification'. The graphical representation of membership function of the RSSI, distance and mobility are in Fig. 2., and Fig. 3. The sender finds the membership function which degree the belongs : $\{$ low, medium, long $\}$, \{low, medium, high\}, \{weak, medium, strong\} and \{VeryBad; Bad; Notacceptable; Acceptable; Good, Ideal\}. By utilizing predefined membership functions and linguistic variables to metamorphose three factors to fuzzy values.

\subsubsection{IF/THEN rules combination and Mapping}

Mapping the fuzzy values for pre-established IF/THEN rules and integrate the rules to obtain the position of the neighbor node as a fuzzy value. The position is Ideal when IF Distance is long, mobility is high, and Signal Strength is Good. In a rule IF part is familiars "precedent" and THEN part is known the consistent. Since multiple rules are applied at a time, combined evaluation results are produced by Min-Max method.

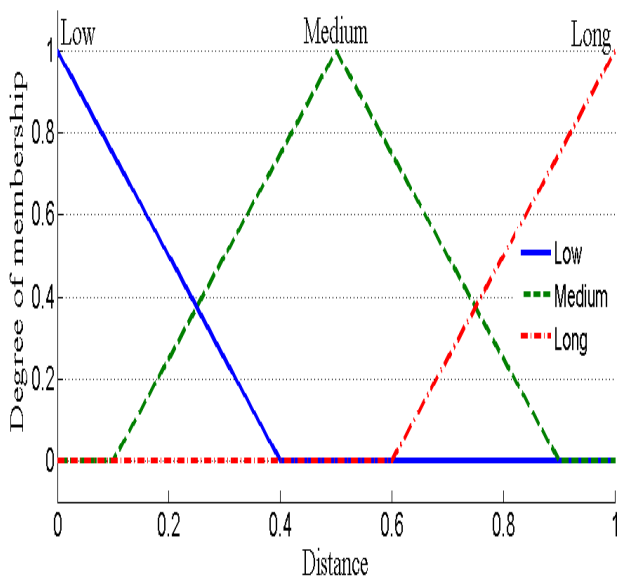

Fig. 2. Distance Factor

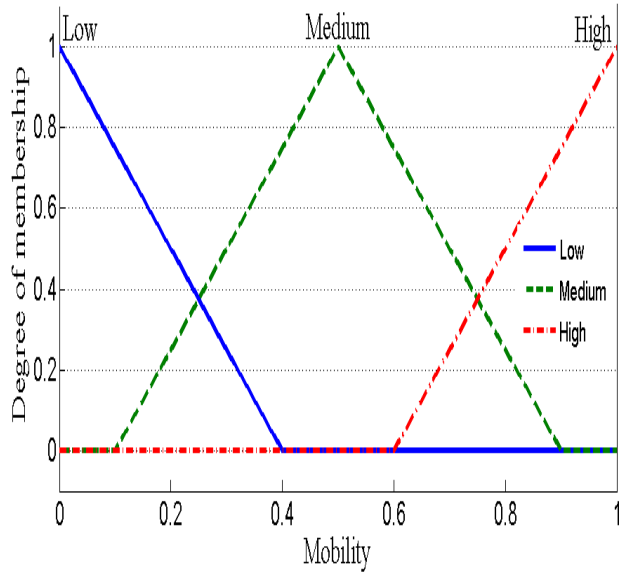

Fig. 3. Mobility Factor

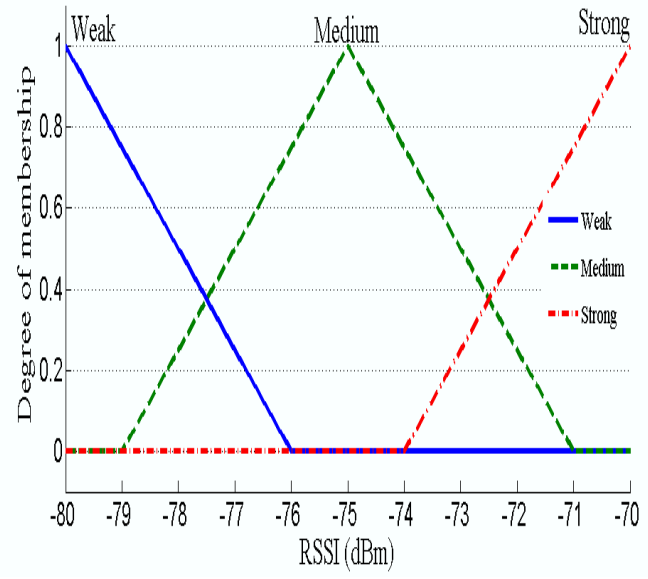

Fig. 4. RSSI Factor

The Fig. 2 represents the $\mathrm{x}$ axis and $\mathrm{y}$ axis which can be taken within the span 0 to 1 . The span of low distance is from 0 to 0.42 . The span of medium distance is 0.1 to 0.92 and the span of long distance is from 0.1 to 1 . The Fig. 2 represents the linguistic variables which are low, medium and long.

The Fig. 3 represents the $\mathrm{x}$ axis and $\mathrm{y}$ axis which can be taken within the span 0 to 1 . The span of low distance is from 0 to 0.42 . The span of medium distance is 0.1 to 0.92 and the span of long distance is from 0.1 to 1 . The Fig. 3 represents the linguistic variables which are low, medium and high. 
The Fig 4 represents the $\mathrm{x}$ axis assigned with RSSI $(-80 \mathrm{dBm}$ to $-70 \mathrm{dBm})$ and $\mathrm{y}$ axis assigned the degree of membership function with 0 to 1 . The values $-79.53 \mathrm{dBm}$ to $-76.01 \mathrm{dBm}$ represent the weak signal, the values $79.10 \mathrm{dBm}$ to $-71 \mathrm{dBm}$ represent the medium signal and strong signal assigned $-74.01 \mathrm{dBm}$ to $-70 \mathrm{dBm}$. The Fig. 4 represents the linguistic variables which are weak, medium and strong.

\begin{tabular}{|c|c|c|c|c|}
\hline Rule No & Distance & Mobility & Signal Strength & Position \\
\hline Rul1 & Long & $\mathrm{H}$ & Strong & Ideal \\
\hline Rul2 & Long & $\mathrm{H}$ & $\mathrm{M}$ & Good \\
\hline Rul3 & Long & $\mathrm{H}$ & Weak & NotAcceptable \\
\hline Rul4 & Long & M & Strong & Good \\
\hline Rul5 & Long & M & $\mathrm{M}$ & Acceptable \\
\hline Rul6 & Long & M & Weak & Bad \\
\hline Rul7 & Long & Low & Strong & NotAcceptable \\
\hline Rul8 & Long & Low & $\mathrm{M}$ & $\mathrm{Bad}$ \\
\hline Rul9 & Long & Low & Weak & VeryBad \\
\hline Rul10 & Medium & $\mathrm{H}$ & Strong & Good \\
\hline Rul11 & Medium & $\mathrm{H}$ & $\mathrm{M}$ & Acceptable \\
\hline Rul12 & Medium & $\mathrm{H}$ & Weak & Bad \\
\hline Rul13 & Medium & M & Strong & Acceptable \\
\hline Rul14 & Medium & M & $\mathrm{M}$ & NotAcceptable \\
\hline Rul15 & Medium & M & Weak & Bad \\
\hline Rul16 & Medium & Low & Strong & $\mathrm{Bad}$ \\
\hline Rul17 & Medium & Low & $\mathrm{M}$ & Bad \\
\hline Rul18 & Medium & Low & Weak & VeryBad \\
\hline Rul19 & Low & $\mathrm{H}$ & Strong & NotAcceptable \\
\hline Rul20 & Low & $\mathrm{H}$ & $\mathrm{M}$ & $\mathrm{Bad}$ \\
\hline Rul21 & Low & $\mathrm{H}$ & Weak & VeryBad \\
\hline Rul22 & Low & M & Strong & Bad \\
\hline Rul23 & Low & M & $\mathrm{M}$ & Bad \\
\hline Rul24 & Low & M & Weak & VeryBad \\
\hline Rul25 & Low & Low & Strong & $\mathrm{Bad}$ \\
\hline Rul26 & Low & Low & $\mathrm{M}$ & VeryBad \\
\hline Rul27 & Low & Low & Weak & VeryBad \\
\hline
\end{tabular}

Table 1. Rules

Where Rul1 to Rul27 represented as Rule1 to Rule27, H is given as High and M is given as Medium.

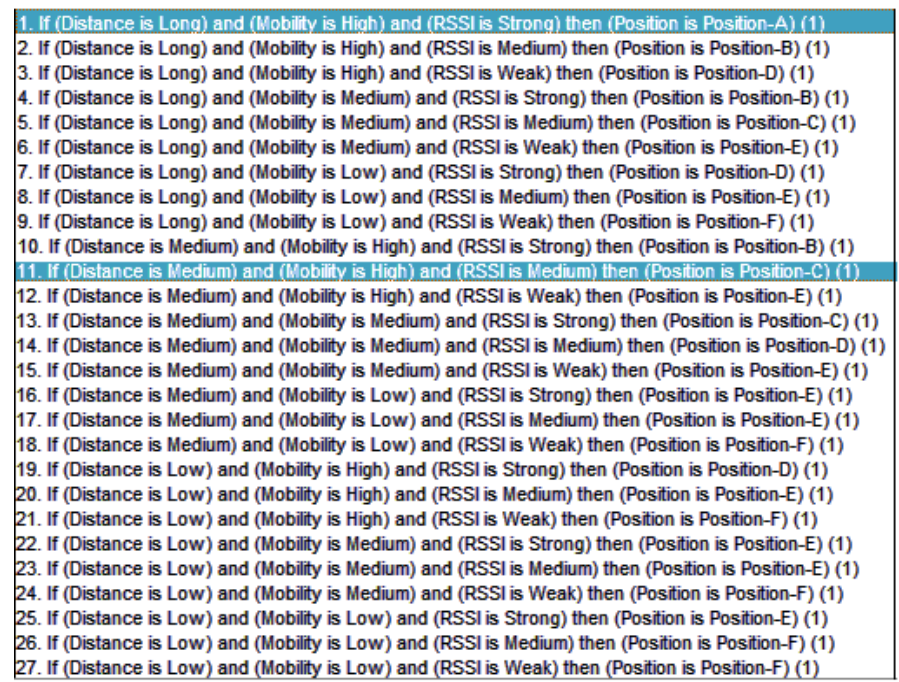

Fig. 5. Rule Editor

The twenty seven possible rules has been shown in table 1. Critical issues that occur further in opting specific forward nodes can be resolved efficiently and traffic modulations are altered with the fuzzy logic based technique. Fig. 5 came from table 1.

\subsection{Plot and Jointly of IF/THEN Rules}

According to three metrics that is vehicle distance, vehicle mobility and vehicle RSSI, by using their fuzzy values a node utilizes the IF/THEN rules (as determined in Table 1 which has 27 fuzzy rules ) to evaluate the position of the neighbor. The related variables of the position are given as \{Ideal, Good, Acceptable, Not Acceptable, Bad and Very Bad). For example from Table 1 Rule1 is represented as follows. IF Distance is long, 
mobility is high, and RSSI is strong THEN position is Ideal. At the same duration, multiple rules are assigned to join their calculation outputs where we utilize the COG (Centre of Gravity) method. In the COG method, for each rule the lowest value of the precedent is employed as the ultimate degree.

\subsection{Fuzzification}

With the help of membership functions, the numerical representation can be changed into fuzzy by using Fuzzification technique. Eqn (1) represent the triangular membership functions that use to form Fig. 2, Fig. 3 and Fig. 4 with the parameters distance, mobility, and RSSI.

$$
\mu_{a}(x i)=\left\{\begin{array}{ccc}
0 & \text { when } & \text { xi } \leq a i \\
\frac{x i-a i}{n i-a i} & \text { when } & \text { ai }<\text { xi }<\text { ni } \\
\frac{\text { bi }-\mathbf{x i}}{\text { bi }-\mathbf{n i}} & \text { when } & \text { ni }<\text { xi }<\text { bi }
\end{array}\right.
$$

A triangular membership function $\mu_{\mathrm{a}}$ (xi) is obtained by the above equation 1 and is depicted in Fig. 6. Crisp output can be obtained from fuzzy inference performance i.e Defuzzification.

The Center of Gravity method (COG) mathematically represented as follows:

$$
X^{\#}=\frac{\int \mu_{a}(x i) \times x d X}{\int \mu_{a}(x i) d X}
$$

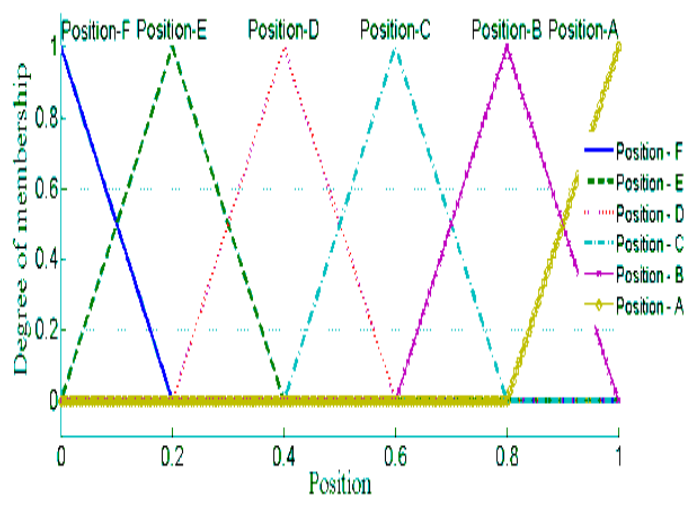

Fig. 6. Membership function Position Output

From Fig. 6 Where Position $\mathrm{A}=$ Ideal, Position $\mathrm{B}=$ Good, Position $\mathrm{C}=$ Acceptance, Position $\mathrm{D}=$ NotAcceptable, Position $\mathrm{E}=$ Bad and Position $\mathrm{F}=$ VeryBad.

\subsection{Defuzzification}

Defuzzification uses output membership function for changing membership degrees to a numeric value as represented in Fig. 6. The defuzzification is done using Center of Gravity (COG). For example the Ideal degree for position is 1 , for position is 0.75 is Good degree and for degree 0.5 is acceptable position and for degree 0.25 is reaming position. The final value is derived from the centroid obtained.

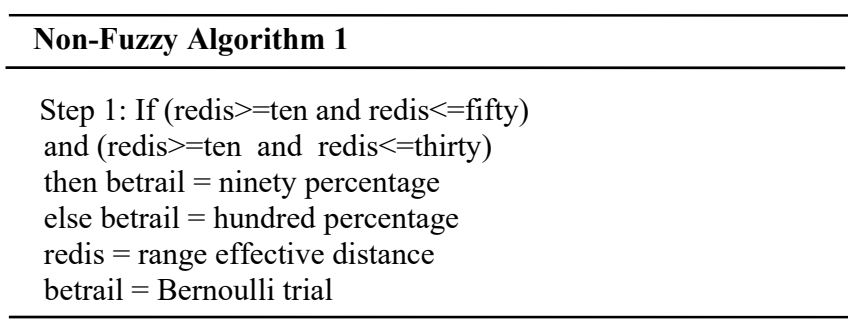


Distance $=290 \mathrm{mts}$

Range is source radio range $=300 \mathrm{mts}$

Range effective distance(redis) $=$ Range- Disatance

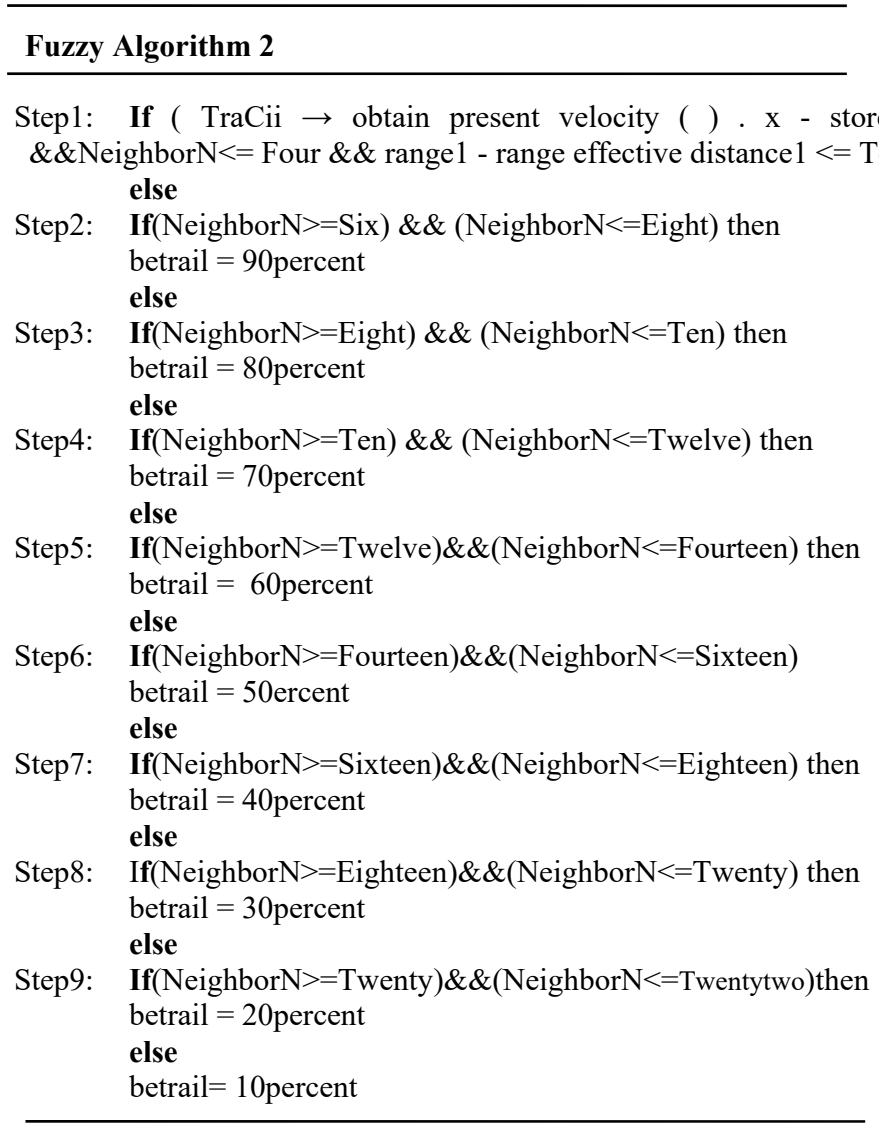

Where betrail $=$ Bernoulli trial

Where NeighborN is Neighbor Nodes.

In non-fuzzy algorithm 1 which vehicle has satisfied with only one condition like long distance, priority is giving to such type of vehicle for receiving emergency message. From fuzzy algorithm 2 Which vehicle has satisfied with more than one condition like long distance and maximum speed and less density, such type of vehicle receive $100 \%$ emergency message that is be trail(Bernoulli trail probability) is $100 \%$, otherwise be trail gets reduced by a factor of $10 \%$.

\section{Performance of Analysis}

\subsection{Distance Factor}

$\operatorname{DFA}(K)=\left\{\begin{array}{cc}\frac{d(K)}{L}, & d(K)<=L \\ 1, & d(K)>L\end{array}\right.$

Up On receiving an emergency message from $\mathrm{K}$, distance factor (DFA) is computed by a node as in equation (3). In Equation (3), $\mathrm{d}(\mathrm{K})$ is distance between occurring node and the node $\mathrm{K}$. By the maximum distance $\mathrm{L}$, consistent communication may be provided.

\subsection{Mobility Factor}

$\operatorname{MFA}(\mathrm{K}) \leftarrow(1-\partial) \times \operatorname{MFA}(\mathrm{K})+\partial \times\left(1-\frac{\left|\mathrm{d}_{\mathrm{t}}(\mathrm{K})-\mathrm{d}_{\mathrm{t}-1}(\mathrm{~K})\right|}{\mathrm{L}}\right)$ 
From a nearby $\mathrm{K}$ up on getting of an emergency message, a node determines a (MFA) mobility factor as in equation (4). For higher MFA value, the compatibility of the surrounding node will be higher. The distance between the surrounding node is $d_{t}(K)$ instant node at a time $t$ and $\partial$ is the smoothing factor in equation (4). By selecting various samples and showed complete analysis.

\subsection{Signal Strength Indicator}

$$
\operatorname{RSSIFA}(\mathrm{K}) \leftarrow(1-\partial) \times \operatorname{RSSIFA}(\mathrm{K})+\partial \times\left(1-\frac{\mathrm{RXThreshd}}{\mathrm{RxPwr}}\right)
$$

After receiving message from a neighborhood node $\mathrm{K}$, it estimates 'Received Signal Strength Indication Factor' (RSSIF) as Eqn.5. 'RxPwr' denoted as received signal power, ' $\partial$ ' is fostering factor, and 'RXThreshd' represents reception threshold.

\section{Simulation Results}

Table 2 specifies the simulation parameters. MATLAB software (R2021a) is used for the simulations of the proposed system. The mobility of nodes are generated by using SUMO tool.

\begin{tabular}{|l|l|}
\hline Environment Measurements & $100 \mathrm{mts} * 100 \mathrm{mts}$ \\
\hline Number of Vehicles & 60 to 400 \\
\hline Mobility Generation & SUMO, OMNeT++ \\
\hline Total Sources & 40 to 50 \\
\hline Total Packets & 50 Packets / Source \\
\hline Packet Size & 512 Bytes \\
\hline Data Rate & One Packet / Sec \\
\hline MAC & IEEE 802.11 MAC (2Mbps) \\
\hline Simulation Time & 500 Secs \\
\hline
\end{tabular}

Table 2. Simulation Parameters

The proposed specifications and the network simulations are briefed in the table 2 . In selection of next forwarder node we can get better presentation in fuzzy logic method, compared to the non-fuzzy method with respect to delay, hops, rebroadcast counts [19-20].

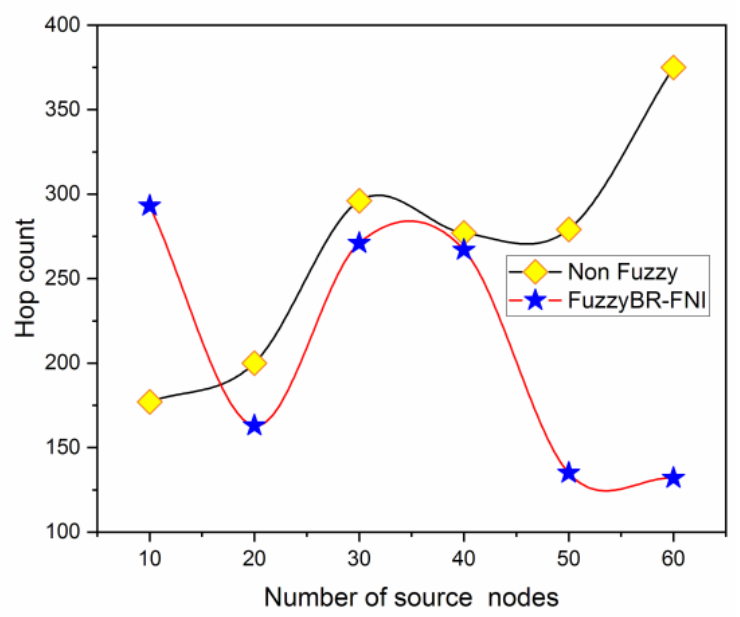

Fig. 7. Number of source nodes vs Hop count. 
The Fig. 7 which depicts the graph between number of source nodes and hop count. It can be noticed that the hop count gets decreases as number of source nodes increases in proposed fuzzy logic technique. In non-fuzzy logic technique, hop count gets increased as number of source nodes increases.

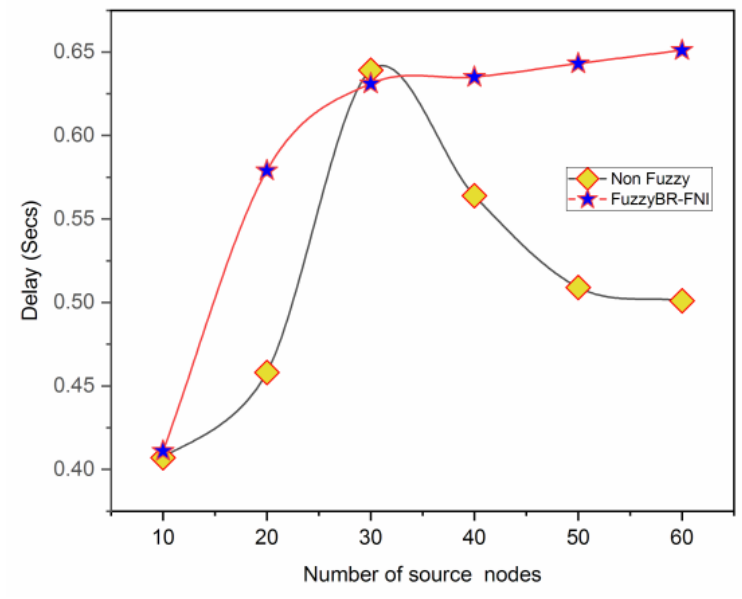

Fig. 8. Delay versus source nodes.

From the Fig. 8, it is observed that when delay increases the number of source nodes also increases in the proposed fuzzy logic technique. In non-fuzzy logic technique delay gets decreased as source nodes number increases.

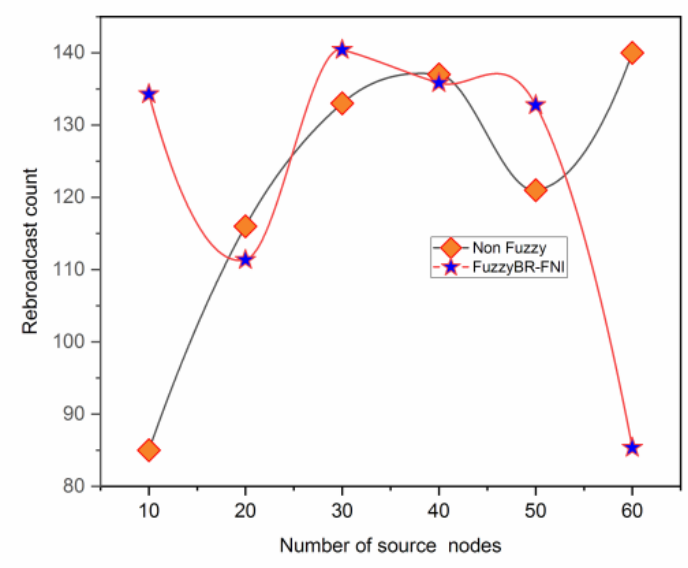

Fig. 9. Rebroadcast count versus source nodes

From the given Fig. 9 it can be represented that as number of source nodes growths and the rebroadcast count reduces in the case of proposed protocol fuzzy logic technique. In non-fuzzy logic technique number of source nodes increases and rebroadcast count gets increased. 


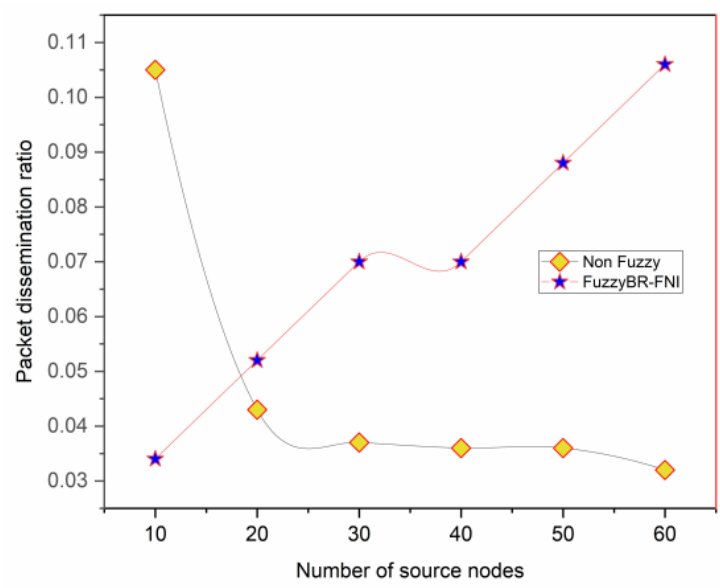

Fig.10. Packet Dissemination Ratio versus source nodes.

From the given Fig. 10 shows the graph between PDR (Packet Dissemination Ratio) and source nodes number. It can be observed that as PDR increases the source nodes number and also increases in proposed protocol fuzzy logic technique. In non-fuzzy logic technique when PDR gets reduced, source nodes number increases.

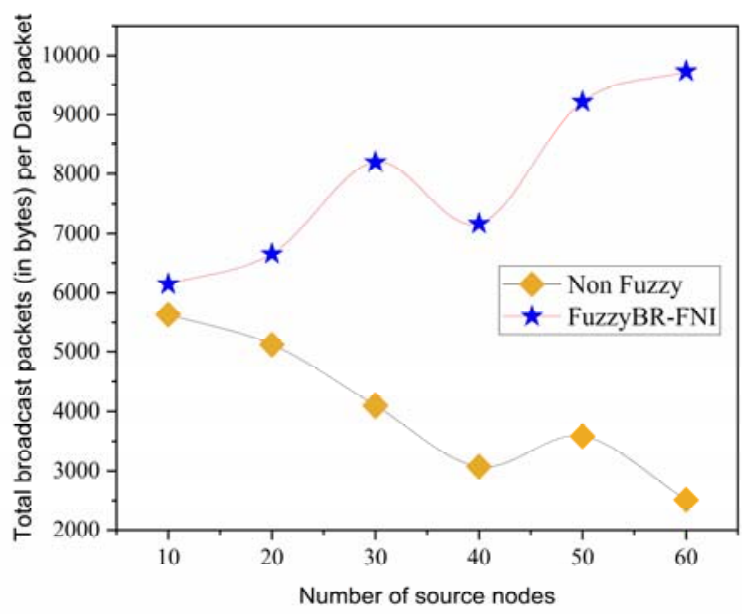

Fig. 11. Source nodes versus Total broadcast bytes per data packet.

From the given Fig. 11 ,it shows that as number of source nodes increases the total broadcast bytes slowly decreases but at node 60, rapidly increases in the case of proposed protocol fuzzy logic technique where as in non-fuzzy logic technique as number of source nodes increases, total broadcast bytes gradually increases at node 50 and at remaining nodes slowly decreases.

\section{Conclusion}

We have developed FuzzyBR-FNI (Fuzzy broadcast with forwarder node information) for VANETs with a several hop broadcast protocol. Proposed protocol is autonomous of placing systems and it surmise the vehicle variation and distance using neighbor information, and three factors (Distance, mobility and RSSI) are used to select best forwarder nodes in VANETs. In VANETs, both simulation and original results revealed that for broadcasting the data dissemination the present protocol can be a practical solution. Simulation results may be persistent about glaring enhancement of the proposed real time methodology on conventional alternatives.

\section{Acknowledgments}

It is an eminent honor to extend my thankfulness to Dr.T.N. Shankar, Prof, Dept of CSE, KLEF, Vaddeshwaram, Guntur, AP, India-522502. For his guidance and incessant help and encouragement in writing this research paper. I got unreserved support from my beautiful family.

\section{References}

[1] N. Wisitpongphan and K.O. Tonguz, "Broadcast storm mitigation techniques in vehicular ad hoc networks," IEEE Wireless Commun, vol.14, no.6, pp.84-94, 2007. 
[2] K. Suriyapaiboomwattana, C. Pornavalai, and G. Chakraborty, "An adaptive alert message dissemination protocol for VANET to improve road safety," IEEE Intl.Conf. on Fuzzy Systems, pp.1639-1644,2009.

[3] M. Slavik and I. Mahgoub, "Stochastic broadcast for VANET," IEEE Consumer Communications and Networking Conference, pp.1$5,2010$.

[4] Y. Mylonas, M. Lestas, and A. Pitsillides, "Speed adaptive probabilistic Flooding in cooperative emergency warning," Proc. $4^{\text {th }}$ Annual Intl. Conf. on wireless Internet, pp. 1-7, Maui, Hawaii, 2008.

[5] M. Bakhouya, J. Gaber, and P. Lorenz, "An Adaptive approach for information dissemination in vehicular and hoc networks, "J. Network and computer Applications, vol.34, pp.1971-1978, 2011.

[6] M. Slavik and I. Mahgoub, "Designing statistical multi-hop wireless broadcast protocols using confidence levels from stochastic models of reachability," Proc. Intl. Wireless Communications and Mobile Computing Conference, pp.1045-1050, 2011.

[7] J. Sahoo, E.H.K. Wu, P.K. Sahu, and M. Gerla, "BPAB: Binary partition assisted emergency broadcast protocol for vehicular ad hoc networks," Proc. 18 $8^{\text {th }}$ Intl. Conf. on Computer Communications and Networks, pp.1-6, San Francisco, USA, 2009.

[8] C. Wu, S. Ohzahata, and t. Kato, "VANET broadcast protocol based on fuzzy logic and lightweight retransmission mechanism," IEICE Trans. Commun, vol.E95-B, no.2, pp.415-425, 2012.

[9] A. Qayyum, L. Viennot, and A. Laouiti, "Multipoint relaying for flooding broadcast messages in mobile wireless networks," $35^{\text {th }}$ Annual Hawaii Intl. Conf. on System Sciences, pp.3866-3875, Big Island, Hawaii, 2002.

[10] L.O. Djedid, N. Lagraa, M. Yagoubi, and K. Tahari, "Adaption of the MCDS broadcasting protocol to VANET safety applications," Intl. Conf. on Innovations in Information Technology, pp.534-538, 2008.

[11] C. Wu, K. Kumekawa, and T,Kato, "A novel multi-hop broadcast protocol for vehicular safety applications," J. Information Processing, vol.18, pp.110-124, 2010.

[12] G.J. Klir, U.S. Clair, and Y. Bo, Fuzzy set theory: Foundations applications, Prentice-Hall, 1997.

[13] The Network Simulator - ns-2, http://www.isi.edu/nsnam/ns/ Accessed on June 23, 2012.

[14] Simulation of Urban Mobility (SUMO), http://sourceforge.net/apps/mediawiki/sumo/index.php, Accessed on June 23, 2012.

[15] D. Krajzewicz, G. Hertkorn, C. Rossel, and P. Wagner, "SUMO (Simulation of Urban Mobility): An open-source traffic simulation," Proc. $4^{\text {th }}$ Middle East Symposium on Simulation and Modelling (MESM2002), SCS European Publishing House, pp.183-187, 2002.

[16] TraNS (Traffic and Network Simulation Environment), http://trans.epfl.ch/ Accessed on June 23, 2012.

[17] S. Karimulla Basha, T. N. Shankar, "Adaptive Probabilistic Broadcast and Safety Contention Window for Progressive Data in VANETs," Journal of Advanced Research in Dynamical and Control Systems (JARDCS), vol. 9, no. 18, pp. 184-196, 2017.

[18] S. Karimulla Basha, T. N. Shankar, "An Efficient Emergency Message Forwarding Technique with Improved Rebroadcast Suppression for VANETs,” International Journal of Engineering \&Technology (IJET), vol. 7, no. 3.1, pp. 1228-1232, 2018.

[19] Basha, S.K., Shankar, T.N. "Fuzzy logic based forwarder selection for efficient data dissemination in VANETs," Wireless Networks, https://doi.org/10.1007/s11276-021-02548-8, 2021.

[20] S. Karimulla Basha, T.N. Shankar, "Fuzzy Based Multi-hop Broadcasting in High-Mobility VANETs," International Journal of Computer Science and Network Security, vol. 21, No. 3, pp. 165-171, 2021.
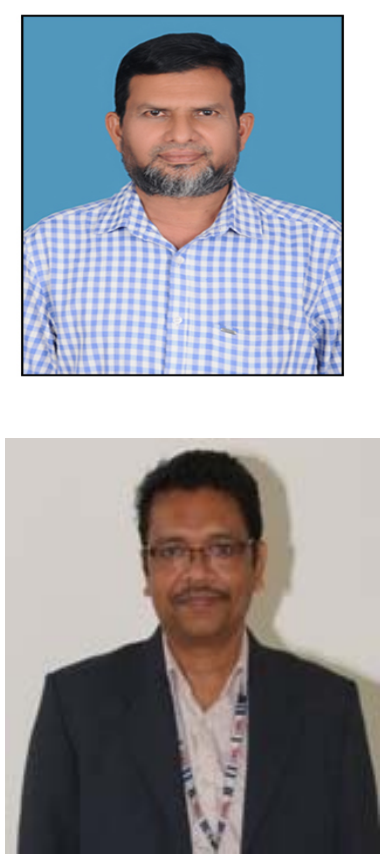

\section{Biography}

S. Karimulla Basha obtained D.E.C.E from Vasavi Polytechnic College, Banaganapalle, AP, India, B.S (Engg Tecnology) from Birla Institute of Technology \& Science, Pilani, Rajasthan, India, M.Tech (CSE) from AITS, Rajampet, Kadapa (Dt), AP, India. At present he is doing Ph.D. in Department of CSE, KL University (KLEF) Vaddeswaram, Guntur, AP, India. under the esteem guidance of T.N. Shankar in Computer Networks in VANETs. He has 4 reputed journals to his credit. His research interests include Vehicular Ad hoc Networks and he is working as the Asst Professor in RGMCET, Nandyal, Kurnool (Dt), AP, India.

T. N. Shankar obtained his M. Tech and a Ph.D. degree in Computer Science \& Engineering from Birla Institute of Technology, Mesra, Ranchi, India. He is the former Asst. Professor, CSE, GMRIT, Rajam, AP, India and Associate Professor, IT, Woldia University, Ethiopia. At present working as Professor in CSE, KL University (KLEF), Vaddeswaram, Guntur, AP, India. He has about 35 reputed journals and 15 conference papers to his credit. His research interests include Information Security and Neural Networks, and he has published a book on neural networks. He is a member of IEEE, ISTE, ACM, and IAENG. 